\title{
Application of nanocomposite cellulose fibers with luminescent properties to paper functionalization
}

\author{
A. Erdman $\cdot$ P. Kulpinski $\cdot$ K. Olejnik
}

Received: 24 December 2015/Accepted: 11 April 2016/Published online: 16 April 2016

(C) The Author(s) 2016. This article is published with open access at Springerlink.com

\begin{abstract}
Cellulose fibres modified with the luminescent inorganic compound were obtained by using $N$-methylmorpholine- $N$-oxide as a direct solvent. The fibres were cut and introduced to the unrefined and refined paper pulp samples. Schopper-Riegler value of the pulps were measured and the laboratory paper samples were formed. Observations under UV radiation showed that the cellulose luminescent fibres were randomly and even scattered in the paper and were well visible. Measurements of the paper properties such as tensile index, Elmendorf tear resistance, Bendtsen air permeability, Bendtsen roughness, folding endurance, bursting strength and tensile energy absorption were carried out and the scanning electron microscopy (SEM) analysis was used for the assessment of the paper structure and the bonding properties of the luminescent fibres. SEM analysis showed that the cellulose man-made fibres create microfibril network and have excellent compatibility with the paper. The results showed that cellulose man-made fibres with luminescent properties express similar properties to natural cellulose fibres (e.g. external fibrillation and bonding properties) which are useful
\end{abstract}

A. Erdman · P. Kulpinski ( $\square)$

Department of Man-Made Fibres, Lodz University of Technology, Zeromskiego 116, 90-924 Lodz, Poland e-mail: Piotr.Kulpinski@p.lodz.pl

K. Olejnik

Institute of Papermaking and Printing, Lodz University of

Technology, Wolczanska 223, 90-924 Lodz, Poland for the purpose of secure paper production. Cellulose man-made fibres introduced to the paper do not have any disadvantageous impact on paper properties.

Keywords Fibres fibrilization - Lyocell - Paper functionalization $\cdot$ Luminescent fibres

\section{Introduction}

Cellulose fibres-both those obtained from vegetable raw materials and the man-made-are widespread materials available in many areas of life. The ongoing development of the methods of modifications of cellulose fibres properties and giving specific functionalities allow finding new uses in many, however not so obvious, applications such as textronic elements (Rubacha and Zieba 2007), electronic component carriers (Alimenti et al. 2015; Steckl 2013), military industry (e.g. paper patching jacketed bullets, filters), and the space industry. The fibres containing in their structure specific chemical compounds with luminescent properties (Kulpinski et al. 2012a) can be effective identifiers of both textile products, paper documents, and those made of plastic. They can also be helpful in management and control of production (Erdman et al. 2016) and transport and storage of products (not only the textile ones). In this case one can speak of the fibres which belong to a class of advanced materials with a possible wide range of 
applications. Moreover, the cellulose fibres are biodegradable and therefore environmentally friendly.

In the research presented in this paper, to prepare the fibres, a method based on $N$-methylmorpholine- $N$ oxide (NMMO) as the direct cellulose solvent was used. Cellulose fibres have been produced using one of the modern and constantly developed textile technologies known as Lyocell technology. Due to the nature of the cellulose solvent used, this technology is environmentally friendly. High flexibility of the manufacturing process of cellulose fibres makes it possible to modify them by introducing into the spinning solution various chemically-different materials that allow obtaining functional fibres with specific properties. In this way cellulose fibres with, for example, electrically conductive and magnetic properties (Liu et al. 2013), bioactive (Kulpinski et al. 2011), sensor fibres (Rubacha 2007), and in recent years also these with luminescent properties were obtained (Kulpinski et al. 2012a). On the basis of previous research it can be concluded that an additional advantage of this technology is the possibility to use modifiers with nanometric size of particles. The combination of advanced technology of producing fibres and nanotechnology offers completely new solutions, and allows producing advanced materials. Due to their chemical similarity to vegetable fibres, the cellulose man-made fibres may also play an important role in functionalization of paper materials. At present there are at least several hundred different types of paper (Soroka 2002) which is due, among other things, to great possibilities of modification of the properties of this material for specific utility needs. These modifications can be carried out both on the level of composition of the paper pulp and paper web manufacturing process, as well as through suitable finishing of the paper web (Holik 2006). In particular, the formation of utility properties of paper during the preparation of a unique formulation of the paper pulp before its manufacturing allows obtaining materials with unique properties that are very difficult to counterfeit. The most commonly used additives include (Scott 1996):

- sizing agents,

- fillers,

- dyes,

- optical brighteners,
- substances improving the strength of the wet paper web,

- substances improving the strength of the dry paper web,

- special additives (e.g. chemicals that cause colour reactions when in contact with certain compounds, natural or synthetic fibres with special properties and others).

In the case of papers that must be uniquely identified (e.g. securities, documents) frequently used additives are synthetic fibres having luminescent properties in a specific wavelength range of electromagnetic radiation. An important factor that determines usefulness of such fibres for use in paper production is their affinity for other components of the paper pulp, particularly natural cellulose fibres. It is well known that plant fibres used in papermaking have a natural tendency of hydrogen bonding between each other (Roberts 1991; Nishiyama et al. 2002). From this point of view, it is primarily about the ability of other fibres, including man-made fibres to make a stable bond with the paper structure-so that during additional processing operations (e.g. paper web rewinding or transferring in a printing machine) the fibres could not be removed from the structure. The fibres with luminescent properties used in modern paper technology are often the synthetic ones therefore have no natural ability to form bonds with natural cellulose fibres. During processing of the paper containing synthetic fibres they are often removed from the paper structure, e.g. as a result of friction when in contact with the transporting rolls. This is a serious technological problem, especially in case of secure papers where the specified number of luminescent fibres per paper unit area is required.

As a result of above problems, the presented research was carried out. The aim of the research was to evaluate the affinity of previously developed luminescent Lyocell type fibres (Kulpinski et al. 2012a) to natural cellulose fibres in terms of their papermaking properties (e.g. their ability to fibrillate, entanglement and bonding). The main criteria determining the usefulness of the fibres were:

- Endurance during papermaking processing (e.g. refining operation),

- The ability to bond with paper structure, 
- Neutral (or positive) impact on the paper strength properties.

On the basis of the recently conducted studies and trials to form cellulose fibres containing different kinds of inorganic modifiers with luminescent properties and on the basis of tests carried out on the properties of the fibres, it was found out that a model fibre that can be used to modify the paper pulp is the cellulose fibre with linear density of 0.6 tex and thickness of $25 \mathrm{~m}$ containing $3 \%$ of modifier $\mathrm{Ce}_{0.85}$ $\mathrm{Tb}_{0.15} \mathrm{~F}_{3}$. This modifier was chosen for the following reasons:

- The method of synthesizing (the form of dispersion in glycerol, which greatly facilitates distribution of the modifier in the spinning solution),

- Particles of the modifier are in nanometric size,

- Sufficiently strong luminescence that allows easy detection of the fibres in both the paper pulp and the final product.

The results presented in this paper are the continuation of previous works (Kulpinski et al. 2012a, b; Kulpinski et al. 2016) which focused only on obtaining the cellulose fibres with various luminescent inorganic modifiers. In the next step the obtained luminescent fibres are introduced to the paper pulp to produce functionalized material.

In this paper the study of morphology and some properties of functional papers modified with cellulose fibres with luminescent properties are presented and the influence of the refining process on the fibres and paper morphology and properties is investigated.

For the assessment of the structure of the paper and the bonding properties of the cellulose luminescent fibres the SEM analysis was used.

The base paper properties were estimated by methods described in the section below.

\section{Materials}

To prepare the cellulose fibres with luminescent properties cellulose pulp (Rayonier) containing $98 \%$ $\alpha$-cellulose with polymerization degree of DP $=1236$ was used. A $50 \%$ aqueous solution of $\mathrm{N}$-methylmorpholine- $N$-oxide (NMMO) manufactured by HUNSTMAN Holland BV, the Netherlands was used as a direct cellulose solvent. Tenox PG [propyl ester of gallic acid; Aldrich (Gillingham, Dorset, UK)] was used as an antioxidant. The modifier of luminescent cellulose fibres cerium fluoride doped with $15 \% \mathrm{~mol}$ of terbium ions with the general formula $\mathrm{Ce}_{0.85} \mathrm{~Tb}_{0.15}$ $\mathrm{F}_{3}$ was prepared in the Department of Rare Earths, Faculty of Chemistry at Adam Mickiewicz University, with the method described in the previous paper (Kulpinski et al. 2016).

Commercial, bleached pine kraft pulp (Sabl) was used for the purpose of laboratory paper production. Polymerization degree of the pulp (DP) was $1081, \alpha-$ cellulose content: $86.6 \%$ and initial moisture content: $93.7 \%$.

\section{Methodology}

Preparation of the cellulose fibres

The cellulose solutions containing $8 \%$ of cellulose were obtained in high efficiency laboratory-scale IKAVISC kneader type MKD 0.6-H60. The mixture of $50 \%$ of water solution of NMMO with cellulose was heated under low pressure and appropriate amount of water was removed from the system. The process was continued until homogeneous transparent solution of cellulose was obtained. The inorganic modifier was added at the beginning of the described process in the form of dispersion in glycerin. The dispersion was added in such amount to obtain $3 \%$ of modifier in the cellulose fibres.

The luminescent fibres were made with the use of dry-wet spinning method on a laboratory-scale piston-spinning device with spinneret equipped with 18 orifices of $0.4 \mathrm{~mm}$ diameter. The fibres were then washed and dried at ambient temperature. The method of obtaining the cellulose fibres was described elsewhere in (Kulpinski 2007; Kulpinski et al. 2012b).

For functionalized paper preparation the cellulose fibres with luminescent properties were cut to the length of $3 \mathrm{~mm} \pm 0.1 \mathrm{~mm}$.

\section{Papermaking}

In order to examine the behaviour of luminescent fibres as an additive in papermaking process, two types of pulps were prepared for laboratory production of paper samples: 
- Refined Sabl pulp with addition of unrefined luminescent fibres,

- Mixture of Sabl pulp and luminescent fibres refined together.

Pulp samples were prepared according to standard ISO 5263-1 and refined in PFI mill according to TAPPI T 248 standard method. The refined pulp was examined and the laboratory sheets of $75 \mathrm{~g} / \mathrm{m}^{2}$ were formed in Rapid-Köthen apparatus according to standard ISO 5259-2:2001. Paper samples were conditioned according to ISO 187:1990 standard. All the properties were tested in accordance with adequate ISO standards:

- SR value (ISO 5267-1:1999),

- Tensile index (ISO 1924-2:2008),

- Elmendorf tear resistance (ISO 1974:1990),

- Bendtsen air permeance (ISO 5636-3:1992),

- Bendtsen roughness (ISO 8791-2:1990),

- Folding endurance (Schopper device-ISO 5626:1993),

- Bursting strength (ISO 2758:2001),

- Tensile Energy Absorption (ISO 12625-4: 2005).

The shape factor was calculated based on the following formula:

$\mathrm{S}_{\mathrm{F}}=(1 / \mathrm{L}) \times 100$

where: L-fibre length measured along the fibre wall, l-length of a straight line between both ends of the fibre

Paper samples with an addition of luminescent fibres were produced from both unrefined and refined pulp. Table 1 gives the number of revolutions used and the corresponding SR values of refined pulp.

In this case, fibres with luminescent properties were introduced to the paper pulp after the refining operation. Luminescent fibres were added to the paper pulp

Table 1 PFI refining conditions and corresponding SR values of refined pulps

\begin{tabular}{ll}
\hline No. of PFI revolutions & SR value $\left({ }^{\circ} \mathrm{SR}\right)$ \\
\hline 0 & 14 \\
2800 & 28 \\
5700 & 44 \\
8600 & 55 \\
\hline
\end{tabular}

in such an amount so that the produced paper contained, respectively: $0 ; 0.5 ; 1$ and $3 \%$ of fibres with luminescent properties. To determine the effect of the refining process on the structure of luminescent fibres one sample of the pulp was refined together with an addition of $3 \%$ cellulose fibres with luminescent properties.

\section{SEM analysis}

The observation of paper surface was done by Scanning Electron Microscope (SEM) VEGA3-SBU and samples of paper were sputtered with gold and palladium (current $18 \mathrm{~mA}$, time $120 \mathrm{~s}$, pressure $4 \mathrm{~Pa}$ ) with the use of 'Mini' Sputter Coater/Glow Discharge System SC7620.

\section{Fibres UV examination}

Identification of luminescent fibres was made using the SLK-UV-IR type UV control lamp by COBRABiD, Poland. Photos of paper samples were taken in UV light at a wavelength of $254 \mathrm{~nm}$.

\section{Results and discussion}

Fibre observations under UV light

Papermaking capabilities of fibres are mainly related to their ability to fibrillate, entangling and binding with other fibres in the way that is similar to natural cellulose fibres. Prior to observations under the electron microscope, the paper samples were subjected to a general identification under UV light where the effect of luminescence was clearly seen (Fig. 1).

In the second phase of the presented research the behaviour of luminescent fibres subjected to the joint refining process together with the Sabl pulp was observed. It was aimed at determining the response of Lyocell type fibres to mechanical forces occurring during stock preparation in the paper technology. This was also interesting due to the fact that during such interactions there may be a disadvantageous release of luminescent substances from the fibre structure. Figure $1 \mathrm{~b}$ shows an image of a sample of paper formed from the pulp refined together with the luminescent fibres observed in UV light. In relation to the fibres 


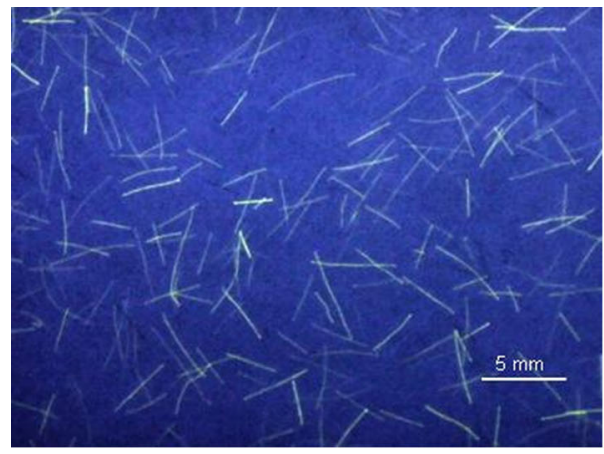

A

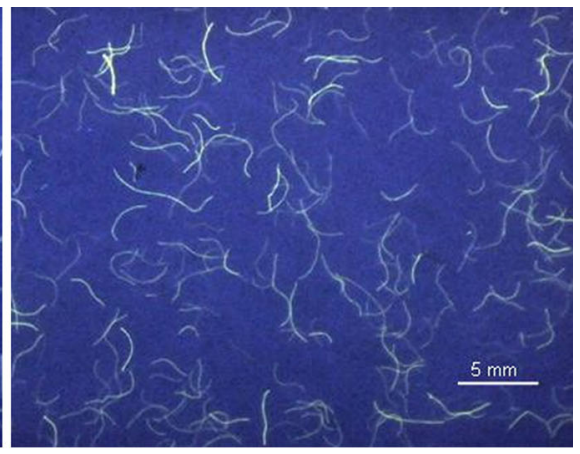

B

Fig. 1 An image of luminescent fibres introduced to the paper structure as seen in UV light (wave length $\lambda=254 \mathrm{~nm}$ ). a Non refined luminescent fibres. b Addition of luminescent fibres subjected to the refining process

shown in Fig. 1a there are clear differences in the shapes of the fibres. In the refining process they were curled and their measured average shape factor $\left(\mathrm{S}_{\mathrm{F}}\right)$ was reduced to the value of approx. 75-80. It was also found out that the fibres had retained the luminescent properties. What is more, although the fibres were subjected to mechanical forces no signs of release of the luminescent substance to the environment were observed.

SEM analysis of papermaking capabilities of luminescent fibres and their affinity to natural cellulosic fibres

Analysing the images obtained by the SEM method (Figs. 2, 3) it can be concluded that cellulose man- made fibres can be easily identified due to their different appearance. Natural cellulose fibres in the paper structure have a form of elongated, flattened objects with uneven surface which is often damaged, especially when the fibrous pulp has been subjected to the refining process. During this process, the cell wall of natural cellulose fibres is fibrillated and partially disintegrated (Karlsson 2006). Fibrils and larger fragments of the cell wall form a complex network of connections with adjacent fibres. Lyocell type fibres with luminescent properties are characterized by a circular cross-section, and their surface is usually smooth, which has been repeatedly confirmed during research on modification of other types of fibres (Kulpinski 2007). The photos of paper samples containing cellulose fibres show that despite all this,
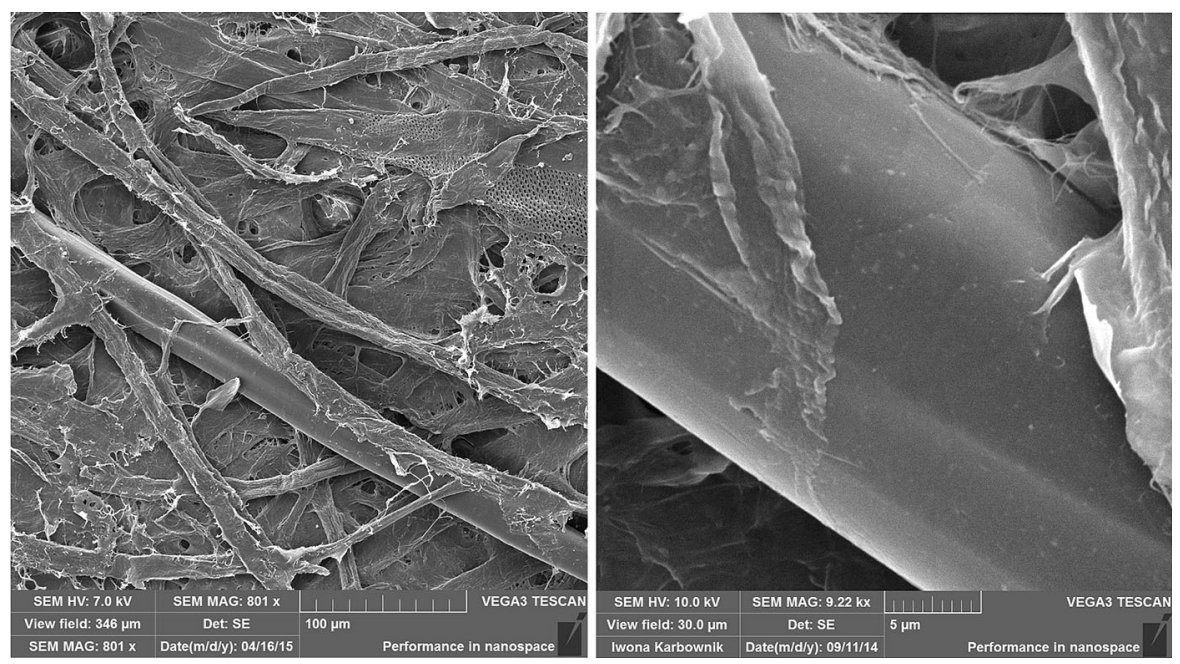

Fig. 2 An image of the Lyocell fiber in the paper structure with a clearly visible bond with natural cellulose fibres of the Sabl pulp 


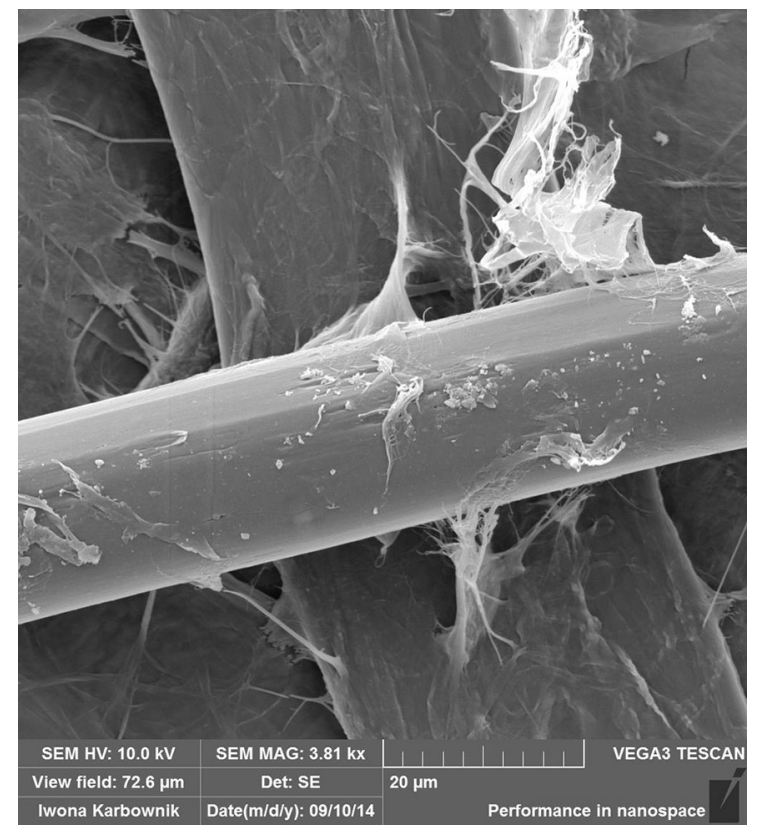

Fig. 3 A fragment of the Lyocell fibre in the paper structure associated with fibrils of natural cellulosic fibre. Visible differences in transverse dimensions of both fibres

the Lyocell type fibres are relatively well bonded in the paper structure. What is interesting, is the fact that the Lyocell type fibres exhibit some characteristics similar to natural cellulose fibres. These characteristics include capacity to self-bonding with other fibres. Although man-made fibres have poorly developed surfaces, and their shape factor $\left(\mathrm{S}_{\mathrm{F}}\right)$ is similar to 100 (which means that in principle they are straight), what can be clearly observed, is the mechanical entanglement with neighbouring objects but also bonding typical of natural fibres (Figs. 2, 3). It can be concluded that these effects are due to the fact that both the Lyocell type fibres and natural cellulose fibres are made from the same polymer type. Cellulose properties and its ability to form hydrogen bonds makes connections of surfaces of the Lyocell type fibres with fibrils durable although, of course, the total bound area will be in this case smaller.

Analysing the images obtained with the SEM method it could be observed that there was damage done to the luminescence fibres caused by the refining process (Figs. 4, 5) and the ways of binding the damaged fibres with other fibres were shown. In Fig. 4 there is a visible part of Lyocell fibre with damaged surface. All cracks in the fibre wall are fully axially oriented-all of them are running along the fibre length. This is an effect derived from the structure of the man-made fibre-the orientation and mutual binding of individual cellulose chains. Nevertheless, this effect can be compared to external fibrillation that occurs during refining of natural cellulose fibres. Under the influence of shear forces in the refining zone, the separation of fragments of the outer layers of the Lyocell fibre occurs. What can also be observed is that those fibril-like objects have the ability to bind with other fibres (Fig. 5). It is clearly visible that the Lyocell fibre binds with the surrounding paper structure both through fibril bands and larger fragments of the cell wall of natural cellulose fibres.

Based on the analysis of the images taken by the SEM method it can be stated that man-made cellulose fibres have a capacity to bind with natural cellulose fibres. It can be also concluded that they have a certain papermaking ability. However, in the absence of a wall of the layered structure (typical to plant cells) Lyocell type cellulose fibres are less able to fibrillate and-as a result-the possibility of their plastic deformation by mechanical forces is significantly lower. Therefore, they will also show lower papermaking potential than natural fibres.

The impact of luminescent Lyocell cellulose fibres on structural and mechanical properties of paper products

In the next part of the research the effect of addition of luminescent fibres on properties of paper products was estimated. The most important structural and strength parameters were determined. The results of all tested paper properties including coefficient of variation (CV) calculated for every specified addition of luminescent fibres are presented in Table 2.

Based on the data presented in Table 2, the Fig. 6 was made showing the effect of addition of luminescent fibres to the Sabl pulp with different degrees of refining on air permeability of the paper samples made from this pulp. On the basis of the measurements taken it was found out that the addition of luminescent fibres had practically no impact on the discussed parameter. The shapes of the curves are basically the same for both the pulp without the addition of the fibres and with the addition of the fibres.

Figure 7 shows the changes of surface roughness of the paper samples made from the cellulose Sabl pulp 

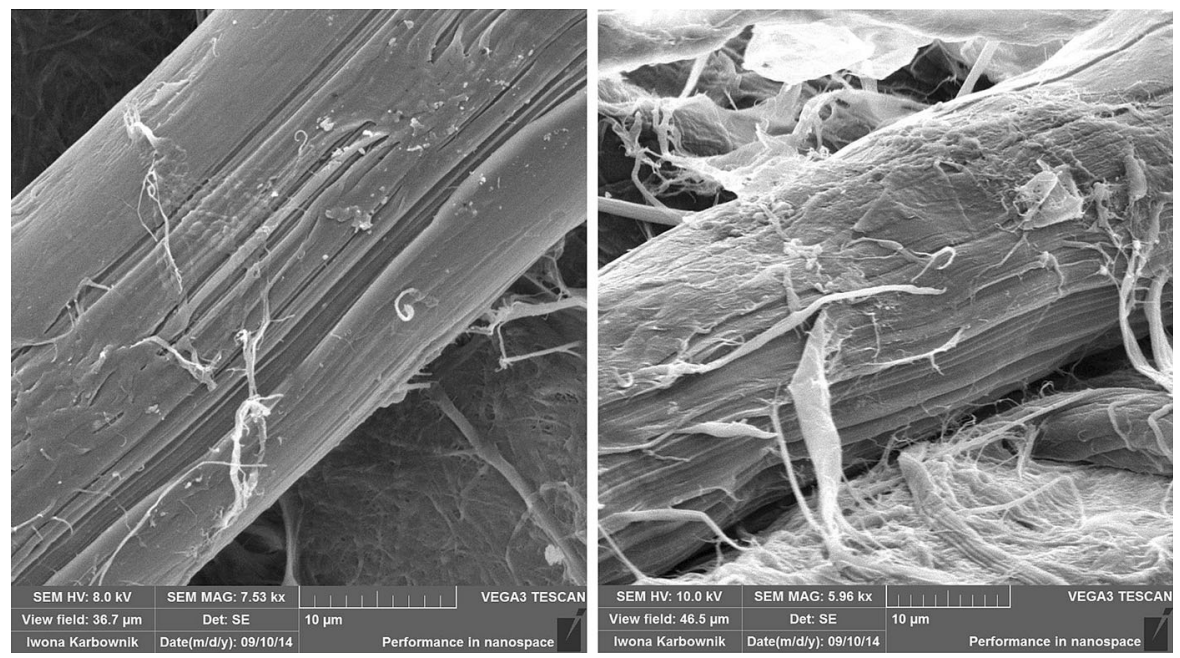

Fig. 4 Enlarged fragments of the Lyocell fibres damaged in the refining process. Noteworthy are the defects that occur mainly in the axial direction of the fibres

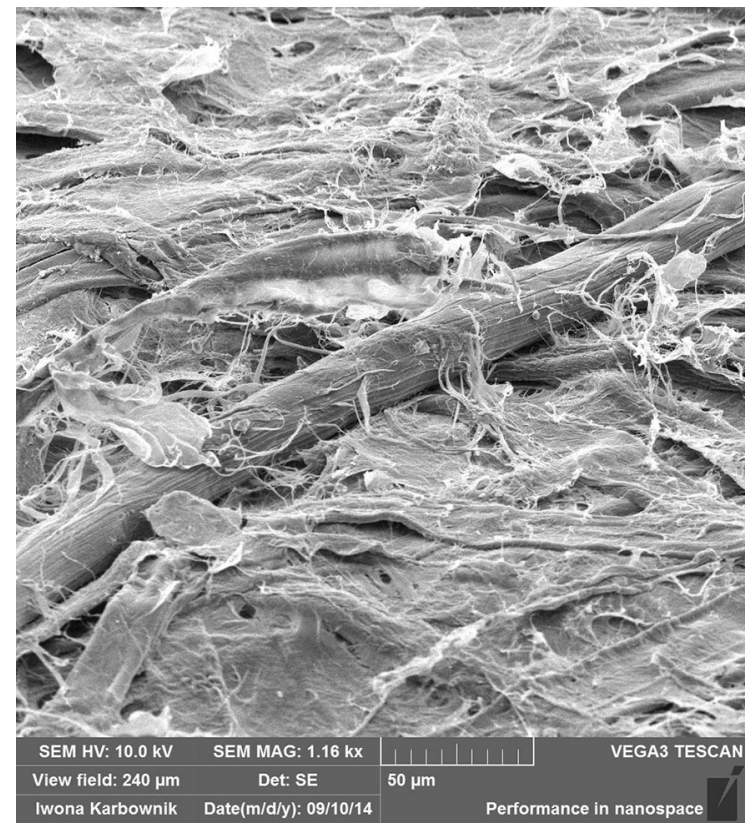

Fig. 5 An image of a Lyocell fibre subjected to the refining process positioned in the paper structure

with different refining degrees and different content of luminescent fibres. It can be concluded that the roughness of the paper surface decreases as the refining time of the paper pulp lengthens. This is due to a progressive homogenization of the fibrous material during the refining process. Based on these results it can be stated that the addition of luminescent fibres in the range of $0.5-3 \%$ did not substantially affect the surface of the paper structure also regardless of the pulp refining degree. The evidence is both the relatively close alignment of experimental points for a given refining degree and the lack of a clear arrangement related to the amount of added luminescent fibres.

Figure 8 shows two opposite mechanical properties of paper i.e. tensile index and tear resistance index. It is well known that during the refining process the tensile index increases while the tear resistance index decreases due to the fact that resistance to tearing is primarily dependent on the length of fibres which are shortened as the refining progresses. So, comparison of those two factors allows us to estimate the changes of the most important mechanical features during refining paper mass. The curves shown in Fig. 8 are linking points of increasing degree of refining (corresponding SR values are shown in Table 1). The obtained results showed that the lower value of tensile index was obtained for the papers without the addition of luminescent fibres. The addition of $0.5-3 \%$ of Lyocell type fibres caused the slight increase in tensile index values and did not decrease the tensile index to tear resistance index ratio. Similar conclusions can be drawn by analysing the changes in the tensile energy absorption (TEA). The results of the changes of the parameter are shown in Fig. 9.

Double folds number (Fig. 10) belongs to one of the most important strength properties of security or 
Table 2 Results of all tested paper properties including coefficient of variation (CV) calculated for every specified addition of luminescent fibres

\begin{tabular}{|c|c|c|c|c|c|c|}
\hline \multirow[t]{2}{*}{ Paper property } & \multirow{2}{*}{$\begin{array}{l}\text { Addition of luminescent } \\
\text { fibres }(\%)\end{array}$} & \multicolumn{4}{|c|}{ No. of PFI revolutions } & \multirow[t]{2}{*}{$\mathrm{CV}(\%)$} \\
\hline & & 0 & 2800 & 5700 & 8600 & \\
\hline \multirow[t]{4}{*}{ Air permeability (ml/min) } & 0 & above 5000 & 1336 & 152 & 15.3 & 6.74 \\
\hline & 0.5 & above 5000 & 2087 & 252 & 46.1 & 6.80 \\
\hline & 1.0 & above 5000 & 1866 & 215 & 29.7 & 5.60 \\
\hline & 3.0 & - & 1862 & 233 & 36.7 & 7.77 \\
\hline \multirow[t]{4}{*}{ Roughness (ml/min) } & 0 & 678 & 376 & 306 & 295 & 16.4 \\
\hline & 0.5 & 573 & 325 & 246 & 243 & 15.2 \\
\hline & 1.0 & 650 & 408 & 268 & 250 & 10.7 \\
\hline & 3.0 & - & 425 & 311 & 300 & 13.6 \\
\hline \multirow[t]{4}{*}{ Tensile index $(\mathrm{N} \times \mathrm{m} / \mathrm{g})$} & 0 & 8.2 & 72.3 & 83.8 & 82.8 & 3.2 \\
\hline & 0.5 & 19.7 & 64.0 & 74.9 & 82.5 & 3.0 \\
\hline & 1.0 & 20.2 & 64.7 & 77.7 & 80.3 & 2.7 \\
\hline & 3.0 & - & 57.8 & 80.8 & 80.3 & 2.6 \\
\hline \multirow[t]{4}{*}{ TEA $(J / g)$} & 0 & 0.139 & 1.717 & 2.001 & 1.948 & 12.8 \\
\hline & 0.5 & 0.216 & 1.562 & 1.720 & 1.824 & 17.5 \\
\hline & 1.0 & 0.139 & 1.550 & 1.840 & 1.850 & 12.8 \\
\hline & 3.0 & - & 1.553 & 1.800 & 1.751 & 11.1 \\
\hline \multirow[t]{4}{*}{ Tearing resistance index $\left(\mathrm{mN} \times \mathrm{m}^{2} / \mathrm{g}\right)$} & 0 & 5.04 & 7.65 & 6.70 & 5.62 & 7.7 \\
\hline & 0.5 & 8.00 & 8.43 & 6.51 & 6.39 & 7.4 \\
\hline & 1.0 & 7.06 & 8.73 & 6.63 & 6.27 & 6.5 \\
\hline & 3.0 & - & 8.41 & 6.37 & 6.20 & 5.3 \\
\hline \multirow[t]{4}{*}{ Double folds number (-) } & 0 & 2 & 1183 & 1136 & 1444 & 20.8 \\
\hline & 0.5 & 6 & 1116 & 1019 & 1059 & 13.8 \\
\hline & 1.0 & 5 & 723 & 936 & 939 & 15.6 \\
\hline & 3.0 & - & 757 & 877 & 888 & 10.5 \\
\hline \multirow[t]{4}{*}{ Burst strength index $\left(\mathrm{kPa} \times \mathrm{m}^{2} / \mathrm{g}\right)$} & 0 & 0.71 & 4.74 & 5.37 & 5.88 & 13.0 \\
\hline & 0.5 & 1.07 & 5.64 & 6.30 & 6.19 & 11.2 \\
\hline & 1.0 & 1.27 & 5.17 & 6.09 & 6.12 & 16.8 \\
\hline & 3.0 & - & 5.38 & 6.00 & 6.04 & 11.6 \\
\hline
\end{tabular}

banknote papers. This test shows the durability of the paper product subjected to repeated folding.

Results in Fig. 10 show that paper made from nonrefined pulp, without the addition of luminescent fibres, had a number of double folds at a level of approx. 1200. As a result of refining an increase in the number of double folds to the final value of approx. 1400 was obtained. The paper made from the pulp containing luminescent fibres is characterized by a lower value of this parameter. For non-refined pulp, with the addition of $3 \%$ of luminescent fibres, the DFN (double fold number) was less than 800 . After joint refining of the Sabl pulp with luminescent fibres an increase in the number of double folds to a value of approx. 890 was obtained. Moreover, based on the obtained results it can be concluded that with the increasing addition of luminescent fibres there is a clear tendency to decrease the number of double folds. This phenomenon may be probably due to different rigidity of natural cellulose pulp fibres, the introduced fibres containing an inorganic modifier, and the lower tendency of Lyocell type fibres to fibrillate resulting in a lower surface bound in the paper structure observed in the microscopic research.

The burst index was the last of the studied strength parameters of the produced paper samples. The results 


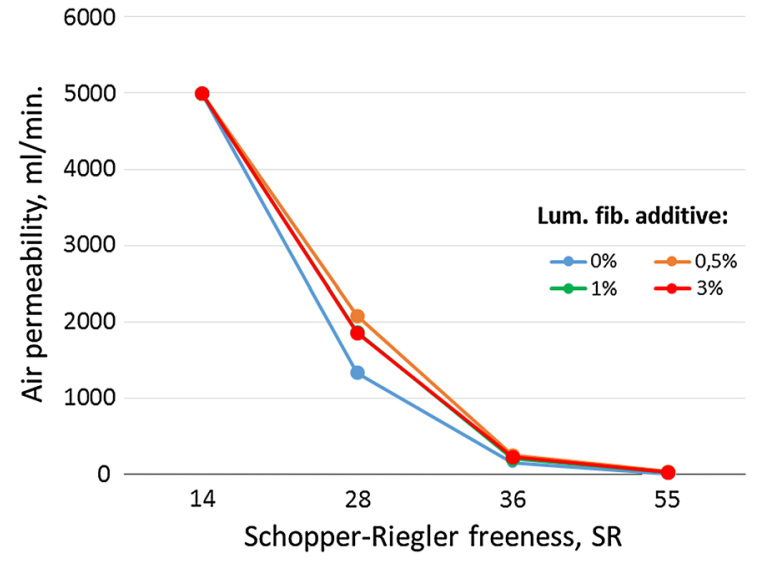

Fig. 6 The influence of addition of luminescent fibres to the pine Sabl pulp with different refining degrees on air permeability of paper samples made from this pulp

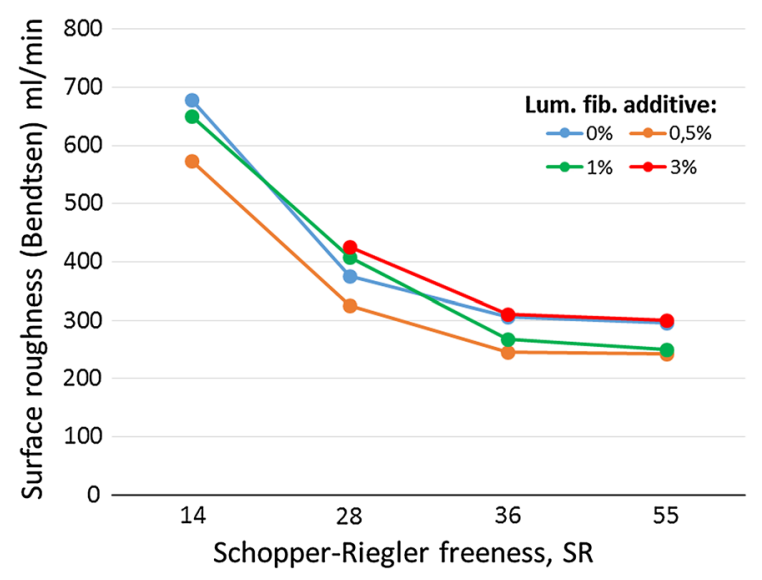

Fig. 7 The influence of addition of luminescent fibres to the pine Sabl pulp with different refining degrees on roughness of the surface of paper samples made from this pulp

presented in Fig. 11 allow to conclude that the luminescent fibres do not have any disadvantageous impact on the discussed parameter.

\section{Conclusion}

Cellulose fibres having an inorganic modifier with luminescent properties were obtained. The fibres cut into 3-mm sections were introduced into the cellulose pulp in the amount of $0.5 ; 1$ and $3 \%$ by weight relative to the weight of the paper pulp. The samples of paper were made from the paper pulp which was refined

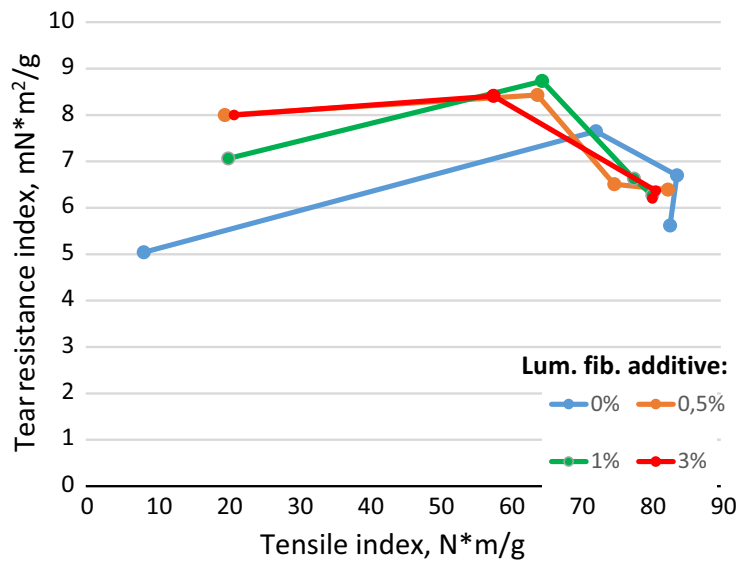

Fig. 8 The comparison of the influence of addition of luminescent fibres for the tensile index to tear resistance index ratio for the paper samples made from pine Sabl pulp of different refining degrees

$$
2,5
$$

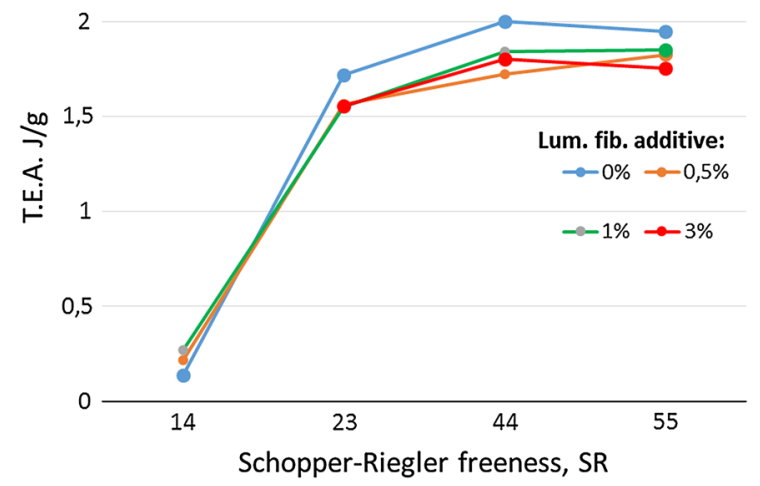

Fig. 9 The influence of addition of luminescent fibres to the pine Sabl pulp with different refining degrees on the TEA indicator of paper samples made from this pulp

together with the Lyocell fibres, and from non-refined pulp.

The analysis of the obtained paper samples with different amounts of man-made cellulose fibres showed that luminescent Lyocell type fibres exhibit a certain similarity to natural cellulose fibres: they can be fibrillated and curled during refining process and they are able to create bonds with natural cellulose fibres. During experiments, Lyocell fibre entanglement similar to natural cellulose fibre entanglement was also observed. The SEM analysis showed that during the refining process Lyocell fibres undergo 


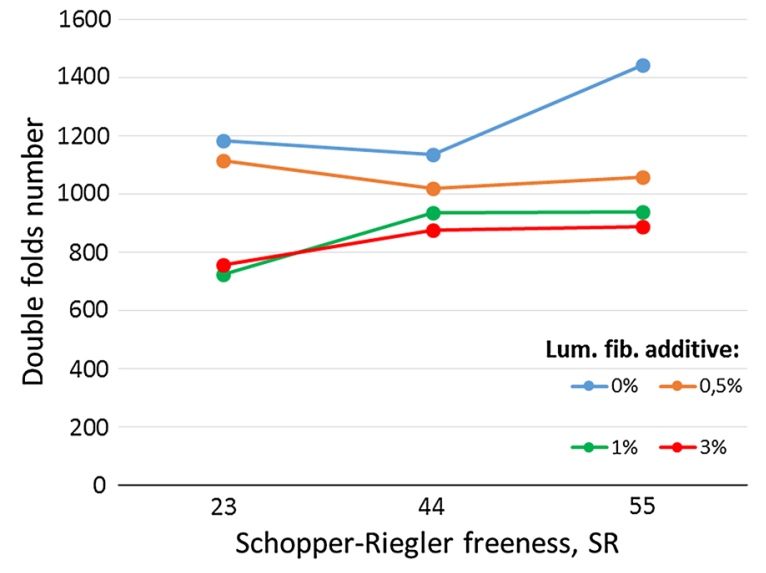

Fig. 10 The influence of addition of luminescent fibres to the pine Sabl pulp with different refining degrees on the number of double folds of paper samples made from this pulp

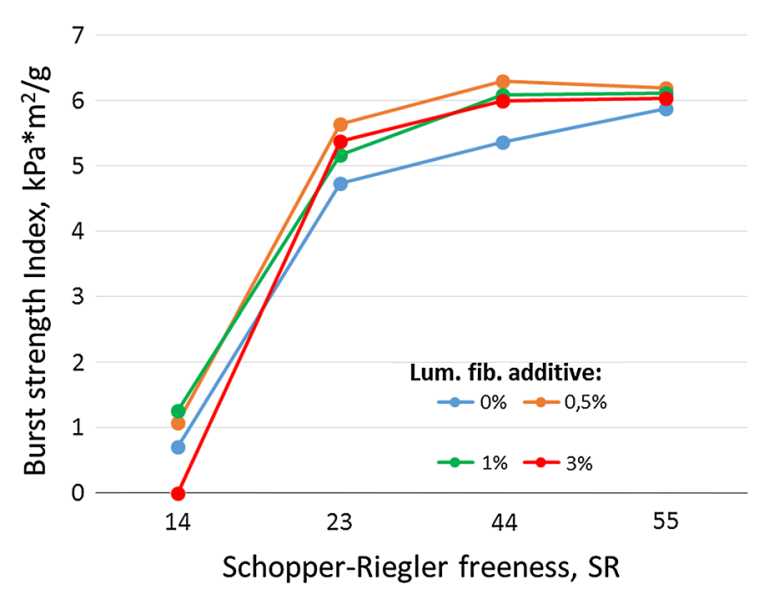

Fig. 11 The influence of addition of luminescent fibres to the pine Sabl pulp with different refining degrees on the burst resistance indicator of paper samples made from this pulp

fibrilization process, which on one hand damages the fibres but on the other hand helps to create bondings with the paper structure by the fibril bands.

It was found out that an inorganic luminescent modifier is permanently bound inside the structure of Lyocell fibres and it cannot be released into the surrounding environment in case of mechanical damage of the fibre (e.g. during pulp refining process).

The research on paper samples containing from 0.5 up to $3 \%$ of addition to luminescent fibres showed no significant changes in both the structural properties (air permeability, surface roughness), and the strength properties. Therefore it can be concluded that Lyocell type fibres have an advantage over other synthetic fibres used in the production of special (e.g. security) papers.

The fibres contain in their structures phosphors usually in the form of nanoparticles evenly distributed inside the fibre structure. Presented results showed that the combination of the technology of production of modifiers with luminescence properties and the process of forming cellulose fibres containing these modifiers allows us to produce a new type of material which can be easily used for security purposes in various types of products, not only the textile ones.

Acknowledgments Financial support from the Polish Ministry of Science and Higher Education; Grant NN 508623 240 is gratefully acknowledged.

Open Access This article is distributed under the terms of the Creative Commons Attribution 4.0 International License (http:// creativecommons.org/licenses/by/4.0/), which permits unrestricted use, distribution, and reproduction in any medium, provided you give appropriate credit to the original author(s) and the source, provide a link to the Creative Commons license, and indicate if changes were made.

\section{References}

Alimenti F, Mariotti Ch, Palazzi V, Virili M, Orecchini G, Mezzanotte P, Roselli L (2015) Communication and sensing circuits on cellulose. J Low Power Electron Appl 5:151-164

Erdman A, Grzyb T, Kulpinski P, Lazarek J, Lis S, Olejnik K, Reczulski M, Szczepaniak PS, Wysocka-Robak A (2016) Estimation of fiber orientation in paper products by image analysis on-line system. Fibres Text East Eur 2(116): $107-112$

Holik H (2006) Handbook of Paper and Board, WILEY-VCH Verlag GmbH \& Co. KGaA, Weinheim, ISBN: 3-52730997-7, 62-126

Karlsson H (2006) Handbook: fibre guide. Fibre analysis and process applications in the pulp and paper industry. Lorentzen \& Wettre, Sweden, ISBN: 91-631-7899-0, 36-39

Kulpinski P (2007) Cellulose fibres modified by hydrophobic type polymer. J Appl Polym Sci 104:398-409

Kulpinski P, Smiechowicz E, Niekraszewicz B, Baciarelli A (2011) Cellulose fibers modified with silver nanoparticles. Cellulose 18:975-985

Kulpinski P, Namyslak M, Grzyb T, Lis S (2012a) Luminescent cellulose fibers activated by $\mathrm{Eu}^{3+}$-doped nanoparticles. Cellulose 19:1271-1278

Kulpinski P, Erdman A, Namyslak M, Fidelus JD (2012b) Cellulose Fibers Modified-by $\mathrm{Eu}^{3+}$-doped Yttria-Stabilized Zirconia Nanoparticles. Cellulose 19:1259-1269 
Kulpinski P, Erdman A, Grzyb T, Lis S (2016) Luminescent cellulose fibers modified with cerium fluoride doped with terbium particles. Polym Comp 37:153-160

Liu S, Luo X, Zhou J (2013). Magnetic responsive cellulose nanocomposites and their applications. In: van de Ven T, Godbout L (eds) Cellulose-medical, pharmaceutical and electronic applications, ISBN 978-953-51-1191-7. doi: 10. 5772/53884

Nishiyama Y, Langan P, Chanzy H (2002) Crystal structure and hydrogen-bonding system in cellulose $\mathrm{I} \beta$ from synchrotron $\mathrm{X}$-ray and neutron fiber diffraction. J Am Chem Soc 124(31):9074-9082
Roberts JC (1991) Paper chemistry. Blackie \& Son, Glasgow Rubacha M (2007) Thermochromic cellulose fibers. Polym Adv Technol 18:323-328

Rubacha M, Zieba J (2007) Magnetic cellulose fibers and their application in textronics. Fibres Text East Eur 15(5-6): 64-65

Scott WE (1996) Principles of wet-end chemistry. TAPPI Press, Atlanta

Soroka W (2002) Fundamentals of packaging technology. IoPP, ISBN 1-930268-25-4

Steckl AJ (2013) Circuits on cellulose. IEEE Spectr 50:48-61 